\title{
Three spacecraft observations of solar wind discontinuities
}

\author{
Timothy S. Horbury, ${ }^{1}$ David Burgess, and Markus Fränz \\ Astronomy Unit, Queen Mary, London \\ Christopher J. Owen \\ Mullard Space Science Laboratory, University College, London
}

\begin{abstract}
Observations of solar wind magnetic field discontinuities using 3 spacecraft allow their orientations to be estimated. During 5 days when Geotail, Wind and IMP 8 were between $6 \times 10^{4}$ and $4 \times 10^{5} \mathrm{~km}$ apart, 35 events identified using the Tsurutani-Smith method were detected in all 3 magnetic field data sets. Normals estimated from interspacecraft timings showed that very few were unambiguous rotational discontinuities, with $77 \%$ likely to be tangential, with $<20 \%$ of the magnetic field at the discontinuity threading the normal plane. However, previous single spacecraft studies using minimum variance suggest that most discontinuities are rotational. Minimum variance analysis resulted in many normal estimates lying far from the timing-derived normals. While some of this discrepancy is likely to be due to random errors in minimum variance vectors, there appears to be a class of events with small field magnitude changes where the minimum variance directions and discontinuity normals are approximately perpendicular, probably due to surface waves on the discontinuities.
\end{abstract}

\section{Introduction}

Early spacecraft measurements of the interplanetary magnetic field revealed the presence of large changes in direction, sometimes accompanied by magnitude changes, over tens of seconds. These events are usually considered to be either rotational (RD) or tangential (TD) MHD discontinuities. RDs and TDs are qualitatively different [Hudson, 1970]: RDs have a field component normal to the plane of the discontinuity and propagate in the plasma frame; TDs do not. RDs are "Alfvénic:" Changes in the magnetic field and plasma velocity across the discontinuity are correlated as for Alfvén waves while field magnitude and density changes are small. TDs separate distinct plasma regions, and can have significant field magnitude and density changes across them.

Estimates of the fraction of TDs vary. Lepping and Behannon [1986] estimated that $50-60 \%$ of discontinuities were TDs, as did Mariani et al. [1973], although Mariani et al. [1983] found around twice as many RDs as TDs, with considerable temporal variation. Smith [1973] used two properties - the field magnitude change across the event and the fraction of the field threading the normal plane - to determine the class of each discontinuity. Using this technique, Neugebauer et al. [1984, hereafter N84] found that $\sim 10 \%$ of events were TDs, although another $30 \%$ were ambiguous. Most of the studies described above are dependent on min-

\footnotetext{
${ }^{1}$ Now at The Blackett Laboratory, Imperial College, London. Copyright 2001 by the American Geophysical Union.
}

Paper number 2000GL000121. 0094-8276/01/2000GL000121\$05.00 imum variance analysis (MVA: Sonnerup and Cahill, 1967) to estimate discontinuity normals, but errors can be large [Sonnerup and Scheible, 1998], particularly when the intermediate to minimum eigenvalue ratio $\left(\lambda_{i n t} / \lambda_{m \imath n}\right)$ is small. A lower limit of 2 is often used [e.g. N84; Lepping and Behannon, 1986], although this can still result in significant errors. $N 84$ considered other parameters when attempting to classify observed events and concluded that ambiguous events showed plasma properties closer to those expected of RDs. However, Denskat and Burlaga [1977] argued using two spacecraft data that TDs could be Alfvénic, as expected of RDs.

To estimate its normal using relative timings between spacecraft, it is necessary to measure a discontinuity at at least three locations. Burlaga and Ness [1969] studied 6 discontinuities measured by Explorers 33, 34, and 35 at separations of 10 to $135 R_{E}\left(1 R_{E}=6378 \mathrm{~km}\right)$ and showed that they were probably TDs in pressure balance with small $\Delta|\mathbf{B}|$. Estimating their normals at the 3 spacecraft using the cross product of up and downstream magnetic fields, Burlaga and Ness [1969] showed that most of the TDs were slightly curved on the spacecraft separation scale.

Recent close encounters between Wind, Geotail and IMP 8 provide a new opportunity to determine the orientations of solar wind discontinuities using this method. We analyse several discontinuities using three spacecraft measurements, compare the results with a minimum variance analysis comparable to that used in earlier work, and discuss similarities and differences between the methods.

\section{Method}

Since 1995, Wind, Geotail and IMP 8 have simultaneously been in the solar wind, a few tens of $R_{E}$ apart, several times: Such encounters typically last 1 or 2 days. We have identified periods when all 3 spacecraft were in the solar wind, unaffected by foreshock activity, with good data coverage, $<70 R_{E}$ apart and containing no obvious large transient events such as CMEs. Such encounters are rare and in this work we consider only 5 such days (days 132 and 133, 1996; days 171, 182 and 183, 1997). Typical inter-spacecraft separations are $\sim 30 R_{E}$ in these events, but range from 9 to $67 \mathrm{R}_{\mathrm{E}}$. We use $3 \mathrm{~s}$ field data from Wind and Geotail and $15 \mathrm{~s}$ data from IMP 8 , with $60 \mathrm{~s}$ plasma velocity data from the Geotail CPI instrument, all in GSE co-ordinates.

We identified discontinuities with the [Tsurutani and Smith, 1979] (hereafter TS) method using $60 \mathrm{~s}$ averaged Geotail field data. Changes in the field were identified as discontinuities if they were sufficiently large compared to the background field and other nearby variations, and of sufficiently short duration. Of such events where data was 


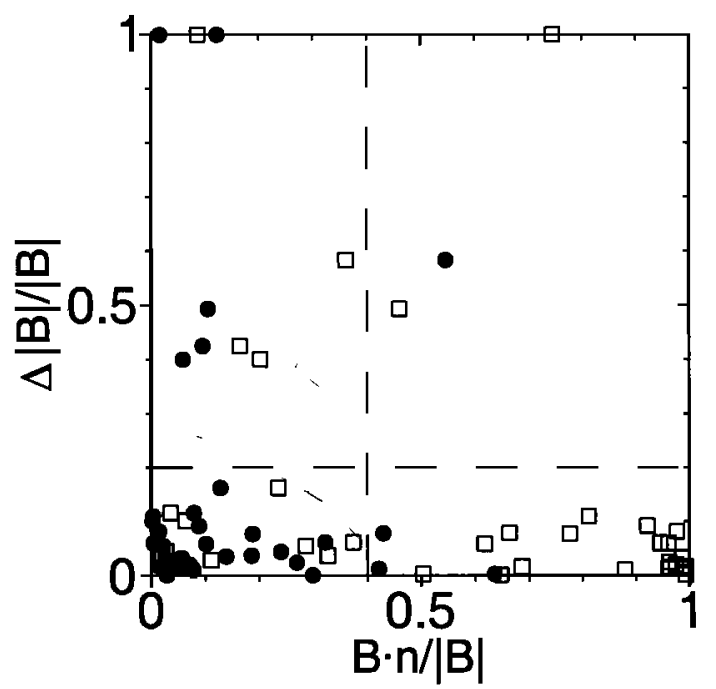

Figure 1. Scatterplot of relative field magnitude change across discontinuities against the fraction of field threading the normal plane. Data are shown based on normal estimates from three spacecraft timings (solid circles) and Wind magnetic field minimum variance vectors (open squares).

available at all 3 spacecraft, the exact time of the event at all 3 spacecraft was identified by eye. Although there were many more discontinuities visible at all 3 spacecraft than were identified by the TS method, we have used it to provide consistency with earlier work.

When a discontinuity was identified, its orientation was estimated using the relative timing of the event in the three data sets. The relative positions of the spacecraft in the plasma frame were calculated at the times of their observations of the event, assuming that they all travelled through the plasma at the bulk velocity measured at Geotail. Each field data point could therefore be located in the plasma frame and the relative positions of the discontinuity measured by the three spacecraft used to calculate its orientation. This method assumes that the discontinuity is stationary in the plasma frame, as discussed later, and that it can be approximated as a plane over the spacecraft separation and there are also other sources of error. Many, such as trajectory and time stamp uncertainties and the assumption of the same plasma velocity at all spacecraft, are small. Others can cause larger errors. The uncertainty in discontinuity timing can be as large as $15 \mathrm{~s}$ in the case of IMP 8 . This corresponds to a typical scale of $\sim 6000 \mathrm{~km}$. Since typical inter-spacecraft separations are $2 \times 10^{5} \mathrm{~km}$, this results in an angular error of $<1^{\circ}$. Overall, we estimate that normal orientations calculated in this work are accurate to $\sim 20^{\circ}$.

\section{Results}

In 5 days of data, the TS method identified 62 discontinuities in Geotail field data when data from all 3 spacecraft were available. Of these, 1 could not be identified by eye in the Geotail data and was discarded, along with 20 which could not be identified at Wind and IMP 8 , and a further 6 on the basis of a minimum variance analysis because $\lambda_{2 n t} / \lambda_{m i n}<2$. Normals of the remaining 35 events were estimated from relative timings as described above.

Magnetic field discontinuities are often classified using the Smith (1973) method, on the basis of the fraction of the field at the event that threads the normal, $\mathbf{B} \cdot \mathbf{n} /|\mathbf{B}|(\mathbf{n}$ is the estimated normal vector) and the relative change in magnitude $\Delta|\mathbf{B}| /|\mathbf{B}|(\Delta|\mathbf{B}|$ is the difference in $|\mathbf{B}|$ averaged over $60 \mathrm{~s}, 60 \mathrm{~s}$ away from the event on either side, and is divided by the larger of these values). Following Smith [1973], N84 identified four classes of event:

$$
\begin{array}{lll}
\text { Rotational(RDs) : } & \mathbf{B} \cdot \mathbf{n} /|\mathbf{B}| \geq 0.4 ; & \Delta|\mathbf{B}| /|\mathbf{B}|<0.2 \\
\text { Tangential(TDs) : } & \mathbf{B} \cdot \mathbf{n} /|\mathbf{B}|<0.4 ; & \Delta|\mathbf{B}| /|\mathbf{B}| \geq 0.2 \\
\text { "Either"(EDs): } & \mathbf{B} \cdot \mathbf{n} /|\mathbf{B}|<0.4 ; & \Delta|\mathbf{B}| /|\mathbf{B}|<0.2 \\
\text { "Neither"(NDs) : } & \mathbf{B} \cdot \mathbf{n} /|\mathbf{B}| \geq 0.4 ; & \Delta|\mathbf{B}| /|\mathbf{B}| \geq 0.2
\end{array}
$$

NDs are inconsistent with MHD RDs or TDs, while EDs could, in principle, be either although with $<10 \%$ or perhaps $20 \%$ of field threading the normal they are more likely to be TDs than RDs. Using single spacecraft MVA estimates of discontinuity normals, N84 found the following percentages for RD:TD:ED:NDs: $61 \%: 7 \%: 30 \%: 2 \%$, typical of other studies. Using normals estimated from 3 spacecraft timings, however, we find the following: $9 \%: 14 \%: 74 \%: 3 \%$, with a much lower fraction of clear RDs and many more EDs. These results are shown in Figure 1 as solid circles. On this basis, most events have very small fields threading their normals: $57 \%$ of events have $<10 \%$, and $77 \%$ have $<20 \%$. These events are consistent, within errors, with TDs.

The large discrepancy between $\mathrm{TD} / \mathrm{RD}$ ratios in this study and previous results [e.g. N84] is striking. It is important to establish whether this difference is a result of the different method used here or if the set of events is statistically different to those in earlier studies. We have therefore performed MVA on each event, and used the classification scheme of $N 84$ using minimum variance vectors as estimates of discontinuity normals: The results are shown in Figure 1 as open squares. Using the same criteria as N84 with Wind MVA estimates, we find the following: $57 \%: 11 \%: 26 \%: 6 \%$, consistent with $N 84$ and some earlier work [e.g Mariani et al., 1983] suggesting that the events used in this work are statistically similar to those used in earlier studies. The discrepancy between $\mathrm{TD} / \mathrm{RD}$ ratios based on normals derived using three spacecraft timings and single spacecraft MVA must therefore be a result of differences in the two methods.

While TDs do not propagate in the plasma frame, RDs travel at the Alfvén speed $\left(V_{A}\right)$ and so normals based on inter-spacecraft timings could be affected by their motion, perhaps making RDs appear to be TDs. To investigate this possibility we assumed that every event was moving at the local $V_{A}$ in 100 random directions. For each velocity, a new

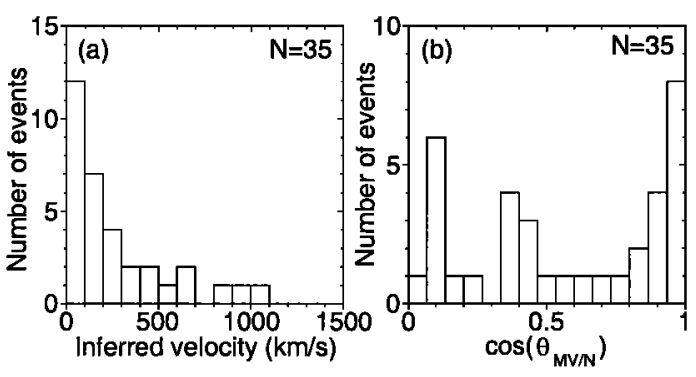

Figure 2. (a) Discontinuity propagation speeds needed to satisfy Geotail-Wind timings, assuming normals aligned with Wind minimum variance vectors. (b) Cosines of angles between the normals estimated from 3 spacecraft timings and those from Wind magnetic field minimum variance vectors. 

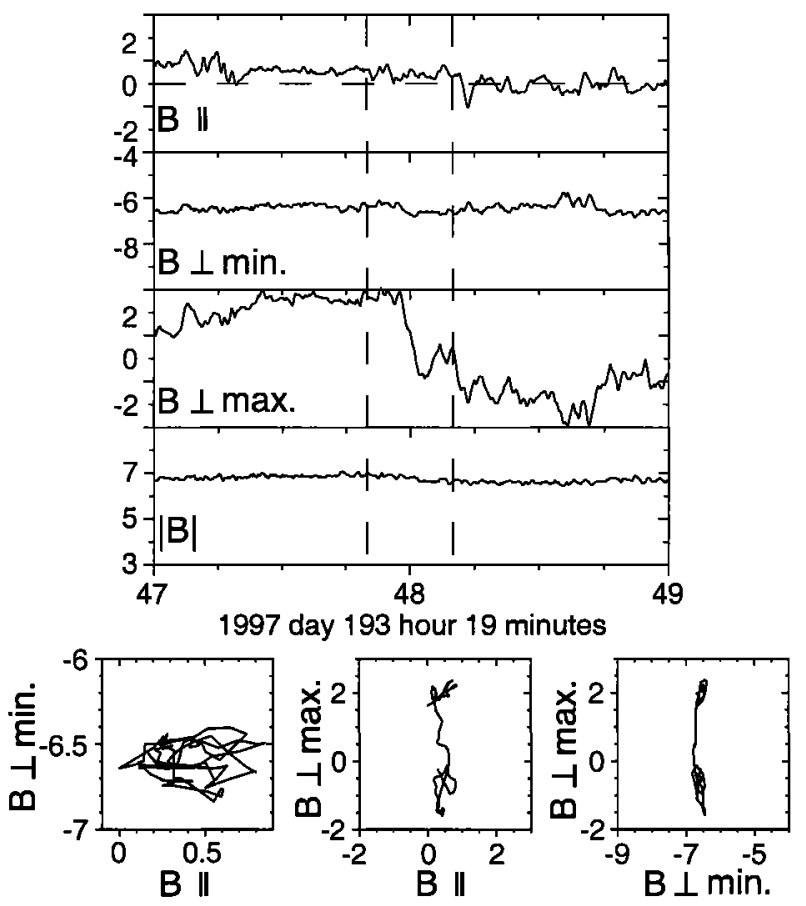

Figure 3. A discontinuity observed at Geotail. Top: 2 mins of $0.25 \mathrm{~s}$ averaged magnetic field data, in a coordinate system with the first axis parallel to the discontinuity normal and the two perpendicular axes selected such that one has a minimum, and one a maximum, variance. Bottom: Hodograms of $20 \mathrm{~s}$ of field data (delineated by vertical lines in the time series) around the event, in the same coordinate system. The left hodogram covers a smaller area than the others.

normal can be calculated from the observed timings, and hence the fraction of the field threading it. Of the 3500 normals estimated in this way, $69 \%$ have $<20 \%$ of the field threading the normal and $48 \%$ have $<10 \%$, similar to statistics assuming no discontinuity motion, and show that these events are still overwhelmingly tangential, and so probably not in motion in the plasma frame. Since this procedure essentially adds $V_{A}$ to the measured plasma speed in many directions, it is equivalent to estimating the impact of errors in the measured plasma velocity on normal estimates using inter-spacecraft timings. Since such errors are likely to be $<20 \mathrm{~km} \mathrm{~s}^{-1}$ and $V_{A} \sim 35 \mathrm{~km} \mathrm{~s}^{-1}$, this shows that velocity errors do not have a significant impact on this method.

Another approach to error estimation is to assume that the MVA-derived normal is correct and find the velocity at which the discontinuity must be moving to satisfy measured 2 spacecraft timings. The deduced speeds of discontinuities using normals from Wind minimum variance vectors and Geotail/Wind timings (Figure 2a) are often very large, with only 5 events $<50 \mathrm{~km} \mathrm{~s}^{-1}\left(\left\langle V_{A}\right\rangle=33 \pm 4 \mathrm{~km} \mathrm{~s}^{-1}\right)$. Therefore, MVA vectors as calculated here do not accurately determine many discontinuity normals, and minimum variance estimates of the relative TD/RD fraction do not reflect true occurrence rates. Now, we compare MVA and 3 spacecraft timing derived normals to determine the cause of this discrepancy, which does not appear to be a function of the ratios of $\lambda_{\max }, \lambda_{\imath n t}$ and $\lambda_{m i n}$, at least for the events considered here where $\lambda_{\imath n t} / \lambda_{m i n}>2$. For example, for the 23 events with ratios between 2 and 5 , the average angle between the minimum variance vector and the timing-derived normal is $49 \pm 6^{\circ}$, while for the 12 events with ratios $>5$, it is $50 \pm 9^{\circ}$. Minimum variance estimates are often consistent between spacecraft $\left(54 \%\right.$ are within $\left.30^{\circ}\right)$ and, while the event sample is small, this agreement appears to be better at higher $\lambda_{\imath n t} / \lambda_{m \imath n}$, as expected.

While angles between normals based on inter-spacecraft timings and those derived from a Wind MVA (Figure 2b) often agree - and in fact all events with $\Delta|\mathbf{B}| /|\mathbf{B}| \geq 0.2$ show good agreement - there is a distinct class where they are almost perpendicular. We consider in detail one such discontinuity, which passed Geotail on day 183, 1997 at 19:48:00.

The top panel of Figure 3 shows 2 mins of $0.25 \mathrm{~s}$ averaged Geotail field data around the event, in a co-ordinate system whose first axis ("B ||") is parallel to the normal derived from inter-spacecraft timings: There is little field in this direction at the discontinuity $(\mathbf{B} \cdot \mathbf{n} /|\mathbf{B}|=0.06)$, as expected for a TD. $|\mathbf{B}|$ is almost constant $(\Delta|\mathbf{B}| /|\mathbf{B}|=0.03)$. The other two axes are chosen such that they have minimum (" $B$ $\perp$ min.") and maximum ("B $\perp$ max.") variance. Hodograms of the field in these co- ordinates are shown at the bottom of Figure 3, for 10s either side of the event (marked by dashed lines in the top panel). Figure 3 shows that the normal direction does not have the lowest variance and so will not be identified as such using minimum variance analysis. Indeed, MVA of 20s of data centred on the event results in the intermediate vector lying within $2^{\circ}$ of the timing-derived normal, while the minimum vector is $88^{\circ}$ from it. The max:int:min eigenvalues are $180: 3.57: 1$ for the 10 s around this event, making the eigenvectors sufficiently well determined according to criteria used in earlier work $[N 84$; Lepping and Behannon, 1986].

The greater variation in the field parallel to the normal than in one direction perpendicular to it results in the minimum variance direction lying nearly perpendicular to the normal. We suggest that this surprising result is due to the presence of surface waves on and around the discontinuity surface. Hollweg [1982] discussed MHD surface waves on TDs and showed that a variety of waves could be supported, but argued that compressive surface waves would be damped, leaving a population of non-compressive modes whose wavevectors - and minimum variance direction - would lie parallel to the mean field, not the normal. The minimum variance direction through the discontinuity discussed here lies within $7^{\circ}$ of the mean field direction: Almost all of the field lies in the "B $\perp$ min." direction (Figure 3 ), which is close to the minimum variance vector, consistent with Hollweg [1982]. Denskat and Burlaga [1977] showed that correlated field and velocity changes occurred across TDs, also consistent with Hollweg [1982] and the waves described by Hollweg have many of the properties observed by N84 at discontinuities which led them to conclude that they were consistent with RDs.

There is more power parallel to the timing-derived normal ("B ||") than in the field-parallel direction (close to "B $\perp$ min."), even well away from the discontinuity. This may be due to the effects of surface waves far from the discontinuity, or, more likely, to field- perpendicular MHD fluctuations [e.g. Belcher and Davns, 1971] unrelated to the event: Power levels far from the discontinuity appear to be similar in both field- perpendicular directions ("B $\|$ " and "B $\perp$ max.").

We find that discontinuities such as that in Figure 3, with small $\Delta|\mathbf{B}|$ and moderate $\left(\sim 30^{\circ}\right)$ angle changes, tend to have minimum variance directions parallel to the mean 
field, not the discontinuity normal. This is a systematic effect, not related to uncertainties in MVA estimates, and applies even within the discontinuity transition. Such events usually have low $\lambda_{2 n t} / \lambda_{m i n}$, but sufficiently high to satisfy the criteria used in earlier studies. We conclude that there is a class of tangential discontinuities with small field magnitude changes that are incorrectly identified by MVA as rotational discontinuities, probably due to the presence of surface waves. This has significant consequences for the classification of discontinuities using single spacecraft data.

Around $35 \%$ of events identified by the TS criterion at Geotail could not be identified at IMP8 and Wind, making it impossible to determine their orientations. This implies that curvature and finite scale sizes are significant on the spacecraft separation scale, as previously shown by Burlaga and Ness [1969]. However, the good agreement between the cross product of magnetic fields up- and downstream of the events, which should lie along the normal of TDs, and the timing derived normals ( $80 \%$ lie within $30^{\circ}$ of each other) suggests that curvature is not significant for the events seen at all 3 spacecraft. There may be a systematic effect causing events seen at Geotail, but not IMP 8 and Wind, more likely to be RDs or TDs than events seen at all 3. For example, RDs could tend to have different orientations or scale sizes than TDs. If all events seen at Geotail but not IMP8 and Wind were RDs, the fraction of TDs (with $\mathbf{B} \cdot \mathbf{n} /|\mathbf{B}|<0.2$ ) would be $49 \%$. However, the TD/RD ratio of those events seen at all 3 spacecraft, based on MVA normals, was similar to that found in earlier, single spacecraft studies (roughly 60\%:10\%:30\%:0\% RD:TD:ED:NS, similar to N84), suggesting that the sample used here is not significantly different from those studies and that there was not a systematic selection effect present. The TD/RD ratio deduced using 3 spacecraft timings is therefore likely to reflect the true occurrence rates in slow solar wind.

\section{Discussion}

On the basis of 3 spacecraft measurements of solar wind discontinuities on scales of around $2 \times 10^{5} \mathrm{~km}$ most ( $>70 \%$ ) appear to be tangential, often with small $\Delta|\mathbf{B}|$ : This result is unlikely to be due to errors in the method. This is in contrast to earlier estimates [e.g. N84] using single spacecraft minimum variance to estimate orientations, which typically found that $\sim 60 \%$ of events were RDs. Although the events described here were recorded in slow solar wind where it is likely that the TD/RD ratio is higher, MVA of the events suggested $57 \%$ were RDs, similar to earlier estimates. Indeed, there is sometimes poor agreement between 3 spacecraft estimates of normals and those deduced from minimum variance. While some of this discrepancy may be due to random errors in the MVA vectors, there appears to be a class of events with small $\Delta|\mathbf{B}|$ where the minimum variance direction is almost perpendicular to the normal, even when the former is well defined on the basis of criteria used in earlier studies [N84; Lepping and Behannon, 1986]. This may be due to surface waves on the discontinuities [Hollweg, 1982] and field-perpendicular fluctuations within and around them, causes which are also consistent with observations of correlated field and velocity variations across TDs.

These results have consequences for the structure of the solar wind. TDs separate distinct plasma regions: A high incidence implies that the solar wind is formed of many such regions with no plasma flow between them and with dramatically reduced energetic particle diffusion. The TDs described here may be the "convected structures" or "2D turbulence" described by Tu and Marsch [1993] and Bieber et al. [1996] and may be responsible for a significant fraction of magnetic field power. This work has only discussed magnetic field data. A future paper will consider changes in parameters such as velocity and density, and a larger set of events, in an attempt better to understand the properties of these structures.

Acknowledgments. This work was supported in part by PPARC(UK) grant GR/L29347. Magnetic field data were provided by $\mathrm{S}$. Kokubun and T. Nagai via DARTS at ISAS (Geotail); R. Lepping, GSFC (Wind); and NSSDC (IMP 8); trajectory data by NSSDC; and Geotail plasma data by U. Iowa.

\section{References}

Belcher, J. W., and L. Davis, Large-amplitude Alfvén waves in the Interplanetary Medium, 2, J. Geophys. Res., 76, 3534$3563,1971$.

Bieber, J. W., W. Wanner, and W. H. Matthaeus, Dominant twodimensional solar wind turbulence with implications for cosmic ray transport, J. Geophys. Res., 101, 2511-2522, 1996.

Burlaga, L. F., and N. F. Ness, Tangential discontinuities in the solar wind, Solar. Phys., 9, 467-477, 1969.

Denskat, K. U., and L. F. Burlaga, Multispacecraft observations of microscale fluctuations in the solar wind, J. Geophys. Res., 82, 2693-2704, 1977.

Hollweg, J. V., Surface waves on solar wind tangential discontinuities, J. Geophys. Res., 87, 8065-8076, 1982.

Hudson, P. D., Discontinuities in an anisotropic plasma and their identification in the solar wind, Planet. Space Sci., 18, 1611$1622,1970$.

Lepping, R. P., and K. W. Behannon, Magnetic field directional discontinuities: characteristics between 0.46 and $1.0 \mathrm{AU}, J$. Geophys. Res., 91, 8725-8741, 1986.

Mariani, F., B. Bavassano, U. Villante, and N. F. Ness, Variations of the occurrence rate of discontinuities in the interplanetary magnetic field, J. Geophys. Res., 78, 8011-8022, 1973.

Mariani, F., B. Bavassano, and U. Villante, A statistical study of MHD discontinuities in the inner solar system, Solar Phys., 83, 349-365, 1983.

Neugebauer, M., D. R. Clay, B. E. Goldstein, B. T. Tsurutani, and R. D. Zwickl, A reexamination of rotational and tangential discontinuities in the solar wind, J. Geophys. Res., 89, 5395$5408,1984$.

Smith, E. J., Identification of interplanetary tangential and rotational discontinuities, J. Geophys. Res., 78, 2054-2063, 1973.

Sonnerup, B. U. Ö., and L. J. Cahill, Magnetopause structure and attitude from Explorer 12 observations, J. Geophys. Res., $72,171-183,1967$.

Sonnerup B. U. Ö., and M. Scheible, Minimum and maximum variance analysis, in Analysis methods for multi-spacecraft data, edited by G. Paschmann and P. W. Daly, ISSI, Bern, 1998.

Tsurutani, B. T., and E. J. Smith, Interplanetary discontinuities: temporal variations and the radial gradient from 1 to $8.5 \mathrm{AU}$, J. Geophys. Res., 84, 2773-2787, 1979.

Tu, C.-Y., and E. Marsch, A model of solar wind fluctuations with two components: Alfvén waves and convective structures, $J$. Geophys. Res., 98, 1257-1276, 1993.

T. S. Horbury, The Blackett Laboratory, Imperial College, London. SW7 2BW U.K. (e-mail: t.horbury@ic.ac.uk)

D. Burgess and M. Fränz, Astronomy Unit, Queen Mary and Westfield College, Mile End Road, London. E1 4NS U.K.

C. J. Owen, Mullard Space Science Laboratory, Holmbury St. Mary, Dorking, Surrey. RH5 6NT U.K.

(Received May 26, 2000; revised August 17, 2000; accepted September 29, 2000.) 